\title{
Stirring Peptide Synthesis to a New Level of Efficiency
}

\author{
Johnny N. Naoum ${ }^{1}$, Israel Alshanski ${ }^{1,2}$, Guy Mayer ${ }^{1,2}$, Poriah Strauss ${ }^{1,2}$, Mattan Hurevich ${ }^{1,2 *}$ \\ ${ }^{1}$ Institute of Chemistry, The Hebrew University of Jerusalem, Edmond Safra Campus, Givat \\ Ram, Jerusalem, 91904, Israel.
}

${ }^{2}$ Center of Nanoscience and Nanotechnology, The Hebrew University of Jerusalem, Edmond Safra Campus, Givat Ram, Jerusalem, 91904, Israel.

*Any correspondence or request should be addressed to Mattan Hurevich. The Hebrew University of Jerusalem. Edmond Safra Campus, Givat Ram, Jerusalem, Israel. Phone: +97226585541. Email: mattan.hurevich@mail.huji.ac.il

\begin{abstract}
Accelerating solid-phase peptide synthesis is crucial to access a large number of peptides and proteins in a short time. Peptide synthesis is usually done using poor mixing methods with slow diffusion of the solid support and reagents, hence the acceleration of the process is achieved by elevated temperature and large reagent excess. In this work, a new setup that relies on fast stirring and heating was used to increase the diffusion of both reagents and solid support. We show that the combination of fast mixing and elevated temperature enables the acceleration of solid-phase peptide synthesis without using a large excess of reagents, providing a greener and accessible alternative to the state-of-the-art.
\end{abstract}

\section{Keywords: SPPS, Peptide synthesis, Fick's second law, Diffusion, Green chemistry}

\section{Introduction}

Solid-phase peptide synthesis (SPPS) is a common methodology for the preparation of peptides. ${ }^{1-3}$ The reactions in this synthetic process are performed in a heterogeneous phase, where the sequence chain extends on a solid support and the reagents are dissolved in an organic solvent. ${ }^{2}$ This enables the removal of the reagents from the growing peptidyl chains by filtration, which reduces the number of purifications. ${ }^{1}$ SPPS, like other processes on insoluble support, is diffusion-dependent. ${ }^{4-6}$ The solid phase process is heterogeneous, hence it obeys Ficks' second law of diffusion (Equation 1). ${ }^{7,8}$ 
Eq. 1: $\quad \frac{d C_{(x, t)}}{d t}=\nabla^{2}\left(D_{(x, T, t)} C_{(x, t)}\right)-k_{(T)} C_{(x, t)}$

Where $\mathrm{C}_{(\mathrm{x}, \mathrm{t})}$ is the reactant concentration, $\mathrm{D}$ is the diffusion parameter, $k$ is the reaction rate coefficient. $T$ is the reaction temperature, $t$ is reaction time, $x$ is the location. $\mathrm{C}_{(x, t)}$ depends on distance and time. $D$ depends on mixing, time, and temperature. $k$ depends on temperature.

For peptide synthesis, both coupling and deprotection follow pseudo-first-order kinetics, as the local concentration of functional groups on the solid phase is very high. ${ }^{9}$ This suggests that both the diffusion and the rate coefficient are detrimental to those reactions. Therefore, the conditions that influence the SPPS reaction efficiency are 1) the initial concentration of reagents; ${ }^{10-12}$ 2) reaction time; ${ }^{12-14} 3$ ) temperature; ${ }^{15,16}$ and 4) the mixing method. ${ }^{2,45}$ Increased reaction durations, high reagent concentrations, and elevation of reaction temperature are commonly applied to accelerate reactions on a solid support. ${ }^{12,17}$ However, the effect of mixing efficiency on SPPS processes is not commonly discussed. ${ }^{4,5,16}$ To perform an efficient SPPS process, one needs to consider all conditions that can affect the parameters related to Fick's second law of diffusion (Equation 1), including mixing, which is setup-dependent.

Different setups are employed for SPPS processes (Fig 1A). The first generations of SPPS setups were based on the rotatory motion of the entire vessel to achieve mixing, due to reports that stirring destroys the integrity of the solid support (Fig 1A). ${ }^{1,218,19}$ The inefficient diffusion in these slow mixing setups is compensated by extending the reactions and/or by increasing the excess of reagents. ${ }^{2,15}$ Microwave-assisted SPPS (Mw-SPPS) which utilizes a stationary reactor with gentle bubbling-based mixing, relies on elevated temperatures for improving reaction efficiency (Fig 1A). ${ }^{20-22} \mathrm{Mw}$-SPPS requires a large excess of reagents in addition to the irradiation and temperature effect for the acceleration of reaction cycles, to compensate for the lack of proper mixing. Flow-based SPPS systems gained much attention in the last decades as they allow automation and acceleration of the process. ${ }^{23-25}$ An automated flow-based setup for SPPS (AFPS) presented an extremely fast peptides and proteins syntheses process. AFPS relies on elevated temperatures for accelerating amide bond formation (coupling) and Fmoc deprotection reactions. In AFPS, a full cycle for the introduction of amino acids to the peptide is performed in a clave of 40 seconds, which is 
extremely fast compared to other setups (Fig 1A). ${ }^{16,17,26}$ In AFPS the solid support is stationary and a large excess of reagents (6-60 equivalents) is used for coupling, proving that elevated temperature and flow-based mixing only partially suffice when the short reactions are employed. The above strategies show that for accelerating SPPS processes in reactors with poor mixing (decreasing $t$, Ficks' law), the use of high concentrations is inevitable (increasing C, Ficks' law).

We recently showed that fast overhead stirring does not break the polystyrene beads, confirming that such setups are viable for SPPS ${ }^{4,5}$ High sheer stirring SPPS (Fig 1A, HSS-SPPS) enabled decreasing the concentration of reagents used for both Fmoc deprotection and amino acid coupling steps, albeit with extended reaction times suggesting that improved mixing by itself is not enough for accelerating SPPS.,5 After realizing the effect of both mixing (HSS-SPPS) and temperature (Mw-SPPS and AFPS), we hypothesized that their combination should enable accelerated SPPS even at low concentrations by maximizing the effect of diffusion.

Here, a new setup that enables both fast stirring and an elevated temperature was developed for the acceleration of SPPS (Fig 1A, High-Temperature Fast Stirring Peptide Synthesis, HTFSPS). The parameters affecting SPPS acceleration in a narrow reactor with overhead stirring were studied based on Fick's second law of diffusion. The influence of stirring, temperature, and concentration on SPPS efficiency in short reaction times was evaluated. HTFSPS was challenged by synthesizing peptides of different lengths and complexity levels, including ones that are prone to fail or epimerize. 

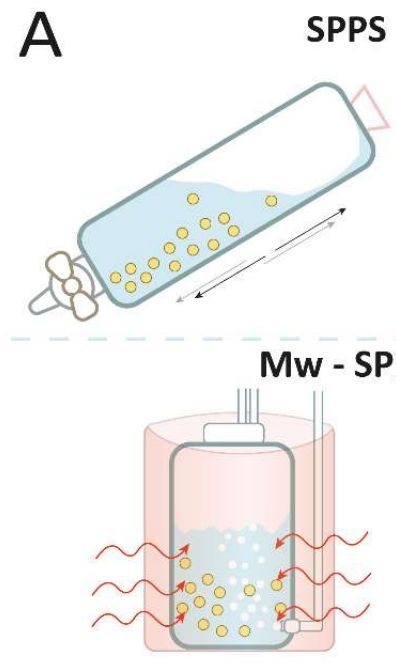

AFPS

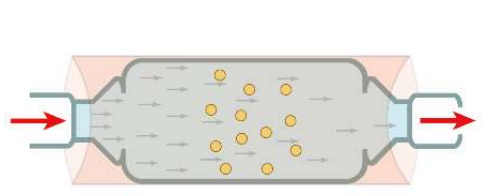

Heating

Mixing

Concentration

Time

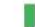

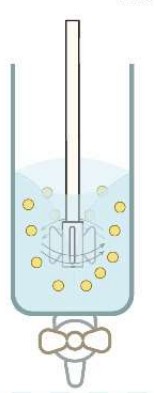

This work: HTFSPS

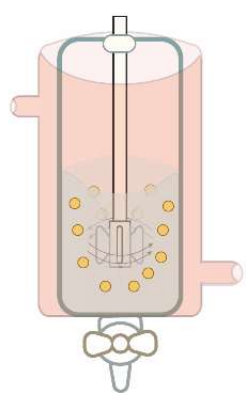

B

(i)

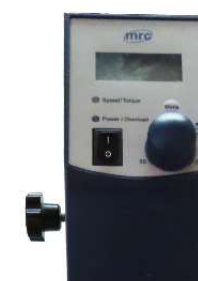

(ii)
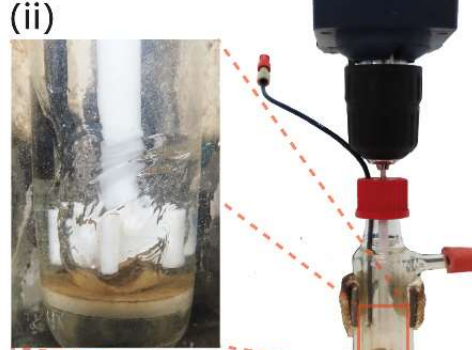

Peptide - a

$\mathrm{H}_{2} \mathrm{~N}$-Lys-Leu-Leu-GIn-Asp-Ile-Leu-Asp-Ala-CONH${ }_{2}$

HFGWI

$\mathrm{H}_{2} \mathrm{~N}$-His*-Phe-Gly-Trp-Ile-CONH $\mathrm{H}_{2}$

HFG

Fmoc-His*-Phe-Gly-CONHH${ }_{2}$

VAS

$\mathrm{H}_{2} \mathrm{~N}$-Cys*-Tyr-Phe-GIn-Asn-Cys*-Pro-Arg-Gly-CONH${ }_{2}$

DDVAS

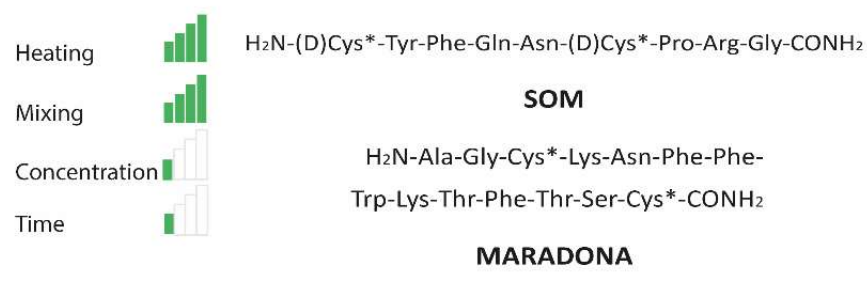

$\mathrm{H}_{2} \mathrm{~N}$-Met-Ala-Arg-Ala-Asp-Orn-Asn-Ala-CONH 2

Figure 1: A) Different setups used for SPPS. B) (i) HTFSPS reactor overview (ii) zoom-in of the impeller region taken using short exposure-time while stirring at $1200 \mathrm{rpm}$. C) The different peptides synthesized in this work. 


\section{Results and Discussion}

A new reactor was designed to enable a full and continuous Fmoc-SPPS process while maintaining high temperature and fast overhead stirring. A self-prepared reactor with a sintered glass filter and a heating jacket was equipped with a feed insert that acts both as a baffle for vortex relapse and as direct delivery of reaction and washing solutions (Fig. 1B, S1, S2). An overhead five-fin turbine agitator PTFE impeller for fast stirring in a narrow dimension was inserted into the reactor. The heating jacket was connected to a circulating water bath. To confirm that this setup does not harm the solid support, microscope images of the beads were taken before and after $7 \mathrm{~h}$ of mixing at $1200 \mathrm{rpm}$ with a temperature of $90{ }^{\circ} \mathrm{C}$ (ESI section 4). The integrity of the beads suggests that the design setup is feasible for SPPS.

\section{Initial evaluation of HTFSPS}

Initial assessment of HTFSPS feasibility was performed by synthesizing a nine-amino acid model peptide-a, KLLQDILDA (Fig. 1) at a constant stirring rate of $1200 \mathrm{rpm}$ using the HTFSPS reactor. ${ }^{16}$ Peptide-a was synthesized in the HTFSPS reactor via several routes, which differ in the reaction conditions, and the crude purity was determined after each synthesis (Fig. 2, Routes 1-3). Reaction mixtures and washing solvents were added to the reactor by injection from the feed line and drained by vacuum filtration. Coupling mixtures containing only two equivalents of protected amino acid, an activator, and a base were added to the reactor without pre-heating or pre-activation. Fmoc deprotection was performed by inserting a solution of $5 \%$ piperidine in DMF without pre-heating. The crude purity of peptide-a synthesized via Route-1 (5 min reactions, $30^{\circ} \mathrm{C}$ ) was above $97 \%$ (Fig 2). This is in line with our previous observation that high shear stirring and long reaction times lead to a high purity. ${ }^{4}$ Furthermore, the result proves that given enough time, an almost complete conversion is achieved in each step when using HSS-SPPS even while using low concentrations of reagents. The crude purity of peptide-a synthesized via Route-2 (30 sec reactions, $30{ }^{\circ} \mathrm{C}$ ) was $91 \%$ (Fig. 2). Since Route-2 applies much shorter reaction times compared to Route-1, it demonstrated that time is a limiting factor in reaching high conversion in each reaction step. Based on Fick's second law, ${ }^{7,8,16,17}$ the temperature was 
elevated to $90{ }^{\circ} \mathrm{C}$ while maintaining short reaction periods, fast stirring, and low reagent concentrations. Peptide-a was synthesized via Route-3 (30 sec reactions, $90{ }^{\circ} \mathrm{C}$ ), which is almost identical to Route- 2 , only that the entire process was performed at $90{ }^{\circ} \mathrm{C}$ instead of $30{ }^{\circ} \mathrm{C}$ (Fig. 2). The crude purity of peptide-a synthesized via Route-3 was above $97 \%$ and took only $27 \mathrm{~min}$ instead of $108 \mathrm{~min}$ via Route-1 with a similar outcome. This suggested that the combination of fast stirring and elevated temperature, enabled by the HTFSPS reactor, can accelerate the SPPS process at low reagent concentrations. Interestingly, although the sequence contains two aspartic acids and the process was performed at a high temperature, ${ }^{17}$ no significant aspartimide formation was observed. We assume that the combination of low piperidine concentration and a short reaction cycle decreases the probability of this side reaction.

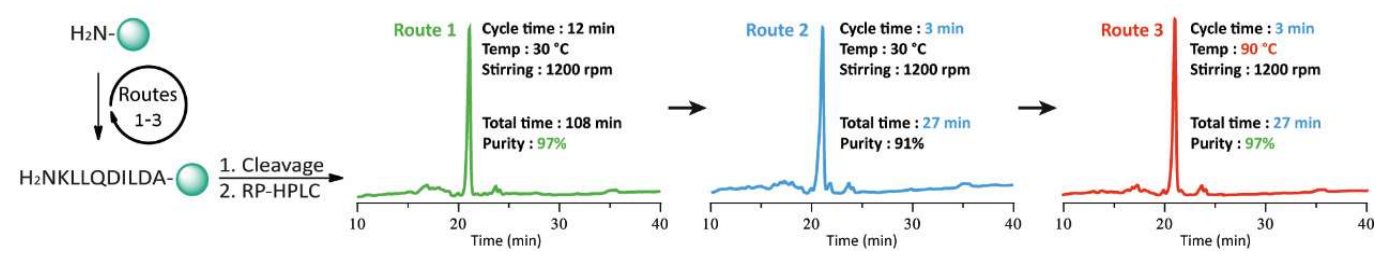

Figure 2: Synthesis of peptide-a at HTFSPS reactor via three different synthetic routes.

To check whether the HTFSPS can be used for difficult couplings, a notoriously hard coupling of Fmoc-L-His(Trt)-OH to the tetrapeptide $\mathrm{H}_{2} \mathrm{~N}$-Phe-Gly-Trp-Ile was used here as a case study (Fig 1, HFGWI). ${ }^{4}$ Coupling of Fmoc-L-His(Trt)-OH was performed in the HTFSPS-reactor using the same conditions described in Route-3, and the HPLC analysis confirmed over $85 \%$ conversion (ESI section 5.4 ). The result is very encouraging, as a similar reaction performed for 60 minutes using $700 \mathrm{rpm}$ stirring at room temperature provided $82 \%$ conversion. ${ }^{4}$ This showed that a combination of elevated temperature and fast stirring can be used instead of extended reaction even for difficult coupling steps, indicating that HTFSPS might surpass other technologies. The joint contribution of elevated temperature and stirring rate in HTFSPS can be used to perform rapid reactions not only for hurdles-free peptide sequences like peptide-a but also for difficult-to-synthesize ones.

\section{HTFSPS does not cause significant epimerization}


It is well accepted that elevated temperatures during coupling reactions can lead to racemization, especially of histidine, and also of cysteine. ${ }^{15}$ To evaluate if HTFSPS results in significant racemization, Fmoc-L-His(Trt)-OH was reacted with the Phe-Gly dipeptide under Route-3 conditions. The HPLC purity of tripeptide Fmoc-HFG and Fmoc-(D)HFG showed epimerization of less than $3 \%$ and $4 \%$, respectively (ESI Sections 5.5-5.7). This specific coupling was chosen since it is prone to epimerization, and is often used as a model case study. ${ }^{15}$ Although the prone-to-epimerize Fmoc-L-His(Trt)-OH was used at a high temperature, the degree of epimerization was not significantly higher than in other methods. Two vasopressin-derived peptides VAS (with two L-Cys) and DDVAS (with two D-Cys) were synthesized using HTFSPS via Route-3 to evaluate epimerization of cysteine. HPLC analysis showed that there were no significant traces of VAS in DDVAS and vice versa (ESI sections 5.8 and 5.9).

These studies indicated that HTFSPS does not result in significant epimerization compared to other methods. ${ }^{16} \mathrm{We}$ assume that the short time and the absence of pre-heating minimize racemization even at elevated temperatures. The above results confirm that peptides containing His and Cys can be synthesized by HTFSPS without using special building blocks or deviating from the standard cycle protocol maintaining the high temperature. ${ }^{17}$

\section{SOM MODEL}

To further push the limits of HTFSPS, the effects of essential parameters (based on Fick's law) were evaluated for the synthesis of a 14-amino acid somatostatin-derived peptide. Somatostatin is an endogenous hormone of the mammalian pituitary gland and is not trivial to synthesize (Fig. 1, SOM sequence). ${ }^{27,28}$ SOM was synthesized here using automated Mw-SPPS at $90{ }^{\circ} \mathrm{C}$ by applying 5 equivalents for couplings periods of at least 2 min (Fig 3, Route-Mw). The Mw-SPPS synthesis afforded SOM in a purity of $41 \%$ indicating that it is a challenging-to-synthesize peptide even using state-of-the-art methods (Fig. 3). Synthesis of SOM by HTFSPS via Route-3 resulted in a crude purity of $60 \%$, which is significantly higher than by Mw-SPPS (Fig 3). This proves that HTFSPS surpasses state-of-the-art Mw-SPPS even when lower concentrations of reagents and shorter reaction times are used at almost the 
same temperature. Since SOM proved such a challenging sequence, it was a suitable candidate for evaluating crucial reaction conditions. Synthesizing SOM at a low temperature via Route-2 did not result in a detectable product (Fig. 3 and ESI section 5.10). Given that the only difference between Route- 2 and Route- 3 is the temperature, it confirms that using $90{ }^{\circ} \mathrm{C}$ is crucial for accelerating the HTFSPS of hard-to-synthesize peptides like SOM. It also shows that evaluating the efficiency only with peptides that are easy to synthesize, e.g., peptide-a, is not sufficient for optimizing a new strategy. To examine the effect of the mixing rate, SOM was synthesized via Route-4, which only differs from Route-3 by employing a stirring rate of $100 \mathrm{rpm}$ instead of $1200 \mathrm{rpm}$ (Fig. 3). Synthesis of SOM via Route-4 resulted in a 48\% purity, which is significantly lower than the purity gains via Route-3, showing that stirring rate is also an important factor in addition to the temperature (Fig. 3). Interestingly, synthesizing SOM at a low stirring rate via Route- 4 was still more beneficial in purity, process time, and reagents quantity, than by Mw-SPPS microwave.
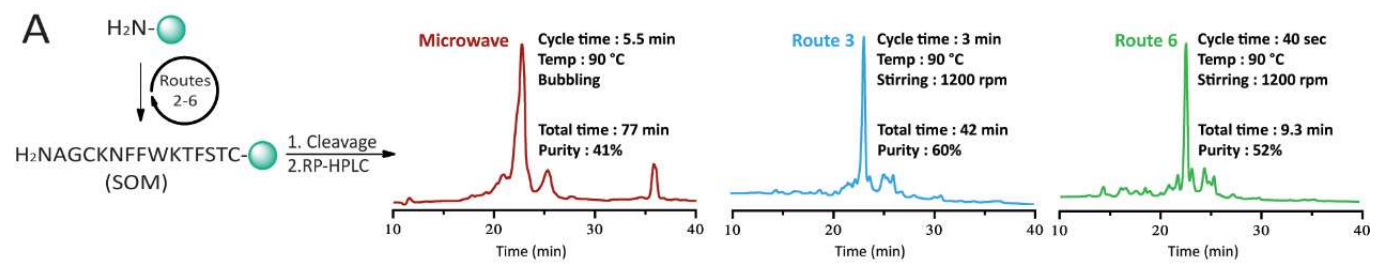

B

\begin{tabular}{|c|c|c|c|c|c|c|}
\hline $\begin{array}{c}\text { SOM } \\
\text { conditions }\end{array}$ & $\begin{array}{c}\text { Mixing } \\
\text { rate }(\mathbf{r p m})\end{array}$ & $\begin{array}{c}\text { Temperature } \\
\left({ }^{\circ} \mathrm{C}\right)\end{array}$ & $\begin{array}{c}\text { Time per } \\
\text { reation }(\mathbf{s e c})\end{array}$ & $\begin{array}{c}\text { Equivalents } \\
\text { of AA and } \\
\text { HATU }\end{array}$ & $\begin{array}{c}\text { Total } \\
\text { synthesis } \\
\text { duration } \\
(\mathbf{m i n})\end{array}$ & Purity (\%) \\
\hline Microwave & 0 & 90 & 120 & 5 & 77 & 41 \\
\hline Route 2 & 1200 & 30 & 30 & 2 & 42 & N.D \\
\hline Route 3 & 1200 & 90 & 30 & 2 & 42 & 60 \\
\hline Route 4 & 100 & 90 & 30 & 2 & 42 & 48 \\
\hline Route 5 & 1200 & 90 & 20 & 6 & 18.7 & 57 \\
\hline Route 6 & 1200 & 90 & 10 & 6 & 9.3 & 52 \\
\hline
\end{tabular}

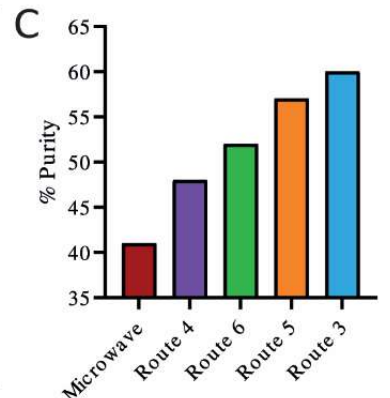

Figure 3: A) analytical HPLC chromatogram of SOM synthesis via Route-Mw, Route-3, and Route-6. B) The different conditions used in the synthesis of SOM. C) Histogram represents the different purities obtained after each synthesis. ${ }^{\text {a }}$ Routes $2-6$ were performed in the HTFSPS reactor.

The above results verify, independently, that the effect of both heating and stirring on SOM synthesis outcome is dramatic. It suggests that fast stirring and a high temperature can be used 
to compensate for low concentration of reagents and/or short the reactions also for peptides that are not easy to synthesize.

After understanding the influence of fast mixing and a high temperature on HTFSPS efficiency, we wanted to check if even shorter reactions are applicable. SOM was synthesized via HTFSPS Route-5 and Route-6, employing short reaction and washings of twenty and ten seconds, respectively. Six equivalents of amino acids and $20 \%$ piperidine were used to compensate for the rapid cycles while the stirring rate, solution volumes, and temperature were identical to Route-3. Synthesis of SOM using Route-5 resulted in a crude purity of $57 \%$, which is very close to the one gained using Route-3 (Fig 3). By performing reaction and washing steps at twenty seconds, the entire SPPS process took $1 / 3$ of the time used for the synthesis via Route-3, whereas the purity was decreased only by 3\%. SOM synthesis following Route- 6 resulted in a further decrease in purity to $52 \%$ (Fig. 3). The synthesis of SOM using Route-6 presents the shortest HTFSPS cycles, as the total time required to introduce each amino acid was 40 seconds, while the payment in purity is reasonable. This proved that a combination of fast stirring, high temperature and slightly increasing the reagent concentrations could compensate for the short reaction time. Comparing SOM synthesis via Route-3 and Route-5 showed that time and concentrations are somehow interchangeable, indicating that all parameters in Ficks' second law must be considered when accelerating SPPS. The reasonable purity obtained in the synthesis of SOM via Route-5 and Route- 6 proved that HTFSPS can be applied for fast synthesis of even difficult-to-synthesize peptides. HTFSPS via Route-6 provided accessibility to peptides in record time, offering a reasonably green and cost-efficient strategy.

To confirm that short cycles can be used for other peptides, we selected a completely random peptide, MARADONA, and synthesized it via Route-6 in a crude purity of above $80 \%$ (ESI section 5.16). This model is the ultimate case study since we had no previous knowledge of the complexity or difficulty of the sequence and it is composed of a variety of canonical and non-canonical amino acids. Route-6 protocol was not optimized for MARADONA, yet the octapeptide was obtained in sufficient purity in a process that took less than ten minutes, thus demonstrating the generality of HTFSPS. 
The above examples highlight the effect of conditions and setup on the SPPS process, in light of Ficks' second law of diffusion. The results indicate that accelerating SPPS can be done by designing a reactor and a process that maximizes the contribution of all parameters and not only by employing a high concentration of reagents. HSS-SPPS and HTFSPS are the only methods reported to date which take advantage of fast overhead mixing (over $600 \mathrm{rpm}$ ) of both reagents and support for improving peptide synthesis processes. Compared to HSS-SPPS, HTFSPS benefits from the contribution of heating which allows acceleration of the process. High temperature increases diffusion and reaction kinetics, but might also result in side reactions like epimerization and aspartimide formation. In the examples shown here, these side reactions seem to be subsided in HTFSPS because of the short reactions, the use of low base concentration, and avoiding preactivation at high temperatures (frequently applied in other systems). This suggests that the process can be performed without changing the temperature between steps, which is a unique and practical advantage over other setups. The ability to decrease reagent excess, shorten reaction time and avoid undesired side reactions benefits directly from the high efficiency of fast overhead stirring. It is important to note that in all processes described above only standard Fmoc protected amino acids were used. In each HTFSPS example, the same activator, base, mixing setup were used for all steps of the synthesis. No special additives, solvents, or amino acid protecting groups were used to avoid side products. Unlike fixed-bed setups, beads swelling and size increase during peptide elongation does not pose a limitation in HTFSPS, hence enabled the use of a high loading resin. Using high-loading resin allowed maximizing the output from each process, using high reagents concentrations without increasing the molar excess and minimizing the volume of solvents.

\section{Conclusion}

Our study proves that both temperature and mixing are crucial for accelerating SPPS. Using large quantities of reagents as a default strategy to accelerate SPPS was replaced here by fast mixing, aiming at generating a high local concentration around the beads at a short time. By

using the HTFSPS reactor, which employs concomitant fast stirring and heating, we shortened the reaction time to seconds. This enabled the synthesis of small-to-medium 
peptides of various levels of complexity within minutes while maintaining low reagent quantities. A green, cost-efficient, and fast SPPS process was developed, which does not require the use of elaborated machinery or a special set of conditions. Performing all steps at a constant temperature and stirring rate is the key for facilitating the process. HTFSPS setup is generic, which ensures that accelerated SPPS can be done at any standard laboratory.

\section{References:}

(1) Merrifield, B. Solid Phase Synthesis. Science (80-. ). 1986, 232 (4748), 341-347.

(2) Barany, G.; Kneib-Cordonier, N.; Mullen, D. G. Solid-Phase Peptide Synthesis: A Silver Anniversary Report. Int. J. Pept. Protein Res. 1987, 30 (1), 705-739.

(3) Albericio, F.; El-faham, A. Choosing the Right Coupling Reagent for Peptides: A Twenty-Five-Year Journey. Org. Process Res. Dev. 2018, 22, 760-772. https://doi.org/10.1021/acs.oprd.8b00159.

(4) Naoum, J.; Alshanski, I.; Gitlin-Domagalska, A.; Bentolila, M.; Gilon, C.; Hurevich, M. Diffusion Enhanced Amide Bond Formation on Solid Support. Org. Process Res. Dev. 2019, 23, 2733-2739. https://doi.org/10.1021/acs.oprd.9b00398.

(5) Alshanski, I.; Bentolila, M.; Gitlin-Domagalska, A.; Zamir, D.; Zorsky, S.; Joubran, S.; Hurevich, M.; Gilon, C. Enhancing the Efficiency of the Solid Phase Peptide Synthesis (SPPS) Process by High Shear Mixing. Org. Process Res. Dev. 2018, 22 (9), 1318-1322. https://doi.org/10.1021/acs.oprd.8b00225.

(6) Tomoi, M.; Ford, W. T. Mechanisms of Polymer-Supported Catalysis, 1. Reaction of 1-Bromooctane with Aqueous Sodium Cyanide Catalyzed by Polystyrene-Bound Benzyltri-n-Butylphosphonium Ion. J. Am. Chem. Soc. 1981, 103 (13), 3821-3828. https://doi.org/10.1021/ja00403a032.

(7) Ogawa, S.; Shiono, N. Generalized Diffusion-Reaction Model for the Low-Field Charge-Buildup Instability at the Si-SiO2 Interface. Phys. Rev. B 1994, 51, 4218 4230 .

(8) Rosenau, P. Reaction and Concentration Dependent Diffusion Model. Phys. Rev. Lett. 2002, 88 (19), 194501. https://doi.org/10.1103/PhysRevLett.88.194501.

(9) Rudzinski, W.; Plazinski, W. Studies of the Kinetics of Solute Adsorption at Solid/Solution Interfaces: On the Possibility of Distinguishing between the Diffusional and the Surface Reaction Kinetic Models by Studying the Pseudo-First-Order Kinetics. J. Phys. Chem. C 2007, 111 (41), 15100-15110. https://doi.org/10.1021/jp073249c.

(10) Kelley, W. S. Therapeutic Peptides: The Devil Is in the Details. Nat. Biotechnol. 1996, 
14 (1), 28-31. https://doi.org/10.1038/nbt0196-28.

(11) Bray, B. L. Large-Scale Manufacture of Peptide Therapeutics by Chemical Synthesis. Nat. Rev. Drug Discov. 2003, 2 (7), 587-593. https://doi.org/10.1038/nrd1133.

(12) Isidro-Llobet, A.; Kenworthy, M. N.; Mukherjee, S.; Kopach, M. E.; Wegner, K.; Gallou, F.; Smith, A. G.; Roschangar, F. Sustainability Challenges in Peptide Synthesis and Purification: From R\&D to Production. J. Org. Chem. 2019, 84 (8), 4615-4628. https://doi.org/10.1021/acs.joc.8b03001.

(13) Goldschmidt Gőz, V.; Nagy, A.; Farkas, V.; Keszei, E.; Perczel, A. Unwanted Hydrolysis or $\alpha / \beta$-Peptide Bond Formation: How Long Should the Rate-Limiting Coupling Step Take? RSC Adv. 2019, 9 (53), 30720-30728. https://doi.org/10.1039/c9ra06124j.

(14) Tofteng, A. P.; Pedersen, S. L.; Staerk, D.; Jensen, K. J. Effect of Residual Water and Microwave Heating on the Half-Life of the Reagents and Reactive Intermediates in Peptide Synthesis. Chem. - A Eur. J. 2012, 18 (29), 9024-9031. https://doi.org/10.1002/chem.201200711.

(15) Hojo, K.; Shinozaki, N.; Hidaka, K. Aqueous Microwave-Assisted Solid-Phase Peptide Synthesis Using Fmoc Strategy . III : Racemization Studies and Water-Based Synthesis of Histidine-Containing Peptides. Amino Acids 2014, 46 (10), 2347-2354. https://doi.org/10.1007/s00726-014-1779-y.

(16) Mijalis, A. J.; Thomas, D. A.; Simon, M. D.; Adamo, A.; Beaumont, R.; Jensen, K. F.; Pentelute, B. L. A Fully Automated Flow-Based Approach for Accelerated Peptide Synthesis. Nat. Chem. Biol. 2017, 13 (5), 464-466. https://doi.org/10.1038/nchembio.2318.

(17) Hartrampf, A. N.; Saebi, A.; Poskus, M.; Gates, Z. P.; Callahan, A. J.; Cowfer, A. E.; Hanna, S.; Antilla, S.; Schissel, C. K.; Quartararo, A. J.; Ye, X.; Mijalis, A. J.; Simon, M. D.; Loas, A.; Liu, S.; Jessen, C.; Nielsen, T. E.; Pentelute, B. L. Synthesis of Proteins by Automated Flow Chemistry. Science (80-. ). 2020, 368, 980-987. https://doi.org/10.26434/chemrxiv.11833503.v1.

(18) Merrifield, R. B. Solid Phase Peptide Synthesis. I. The Synthesis of a Tetrapeptide. J. Am. Chem. Soc. 1963, 85 (14), 2149-2154. https://doi.org/10.1021/ja00897a025.

(19) Lukas, T. J.; Prystowsky, M. B.; Erickson, B. W. Solid-Phase Peptide Synthesis under Continuous-Flow Conditions. Proc. Natl. Acad. Sci. U. S. A. 1981, 78 (5), 2791-2795. https://doi.org/10.1073/pnas.78.5.2791.

(20) Jaradat, D. M. M. Thirteen Decades of Peptide Synthesis: Key Developments in Solid Phase Peptide Synthesis and Amide Bond Formation Utilized in Peptide Ligation. Amino Acids 2018, 50 (1), 39-68. https://doi.org/10.1007/s00726-017-2516-0. 
(21) Pedersen, S. L.; Tofteng, A. P.; Malik, L.; Jensen, K. J. Microwave Heating in Solid-Phase Peptide Synthesis. Chem. Soc. Rev. 2012, 41 (5), 1826-1844.

https://doi.org/10.1039/c1cs15214a.

(22) Galanis, A. S.; Albericio, F.; Grøtli, M. Solid-Phase Peptide Synthesis in Water Using Microwave-Assisted Heating. Org. Lett. 2009, 11 (20), 4488-4491.

https://doi.org/10.1021/o1901893p.

(23) Flögel, O.; Codée, J. D. C.; Seebach, D.; Seeberger, P. H. Microreactor Synthesis of B-Peptides. Angew. Chemie - Int. Ed. 2006, 45 (42), 7000-7003.

https://doi.org/10.1002/anie.200602167.

(24) Wang, W.; Huang, Y.; Liu, J.; Xie, Y.; Zhao, R.; Xiong, S.; Liu, G.; Chen, Y.; Ma, H. Integrated SPPS on Continuous-Flow Radial Microfluidic Chip. Lab Chip 2011, 11 (5), 929-935. https://doi.org/10.1039/c0lc00542h.

(25) Sletten, E. T.; Danglad-Flores, J.; Nuño, M.; Guthrie, D.; Seeberger, P. H.; Seeberger, P. H. Automated Glycan Assembly in a Variable-Bed Flow Reactor Provides Insights into Oligosaccharide-Resin Interactions. Org. Lett. 2020, 22 (11), 4213-4216.

https://doi.org/10.1021/acs.orglett.0c01264.

(26) Mohapatra, S.; Hartrampf, N.; Poskus, M.; Loas, A.; Gómez-Bombarelli, R.; Pentelute, B. L. Deep Learning for Prediction and Optimization of Fast-Flow Peptide Synthesis. ACS Cent. Sci. 2020, 6 (12), 2277-2286. https://doi.org/10.1021/acscentsci.0c00979.

(27) Rivier, J.; Kaiser, R.; Galyean, R. Solid-Phase Synthesis of Somatostatin and Glucagon-Selective Analogs in Gram Quantities. Biopolymers 1978, 17, 1927-1938.

(28) Modlin, I. M.; Pavel, M.; Kidd, M.; Gustafsson, B. I. Review Article: Somatostatin Analogues in the Treatment of Gastroenteropancreatic Neuroendocrine (Carcinoid) Tumours. Aliment. Pharmacol. Ther. 2010, 31 (2), 169-188. https://doi.org/10.1111/j.1365-2036.2009.04174.x. 